Comparisons of the DSPEC and DSPEC Plus Spectrometer Systems

Duc T. Vo 



\section{DISCLAIMER}

This report was prepared as an account of work sponsored by an agency of the United States Government. Neither the United States Government nor any agency thereof, nor any of their employees, make any warranty, express or implied, or assumes any legal liability or responsibility for the accuracy, completeness, or usefulness of any information, apparatus, product, or process disclosed, or represents that its use would not infringe privately owned rights. Reference herein to any specific commercial product, process, or service by trade name, trademark, manufacturer, or otherwise does not necessarily constitute or imply its endorsement, recommendation, or favoring by the United States Government or any agency thereof. The views and opinions of authors expressed herein do not necessarily state or reflect those of the United States Government or any agency thereof. 


\section{DISCLAIMER}

Portions of this document may be illegible in electronic image products. Images are produced from the best available original document. 


\section{CONTENTS}

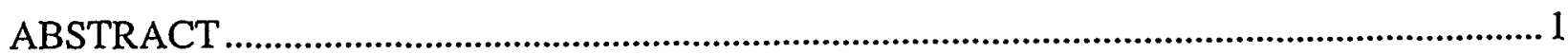

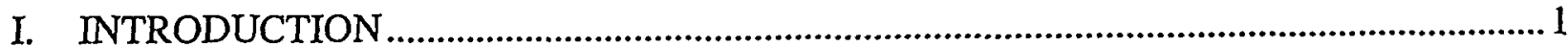

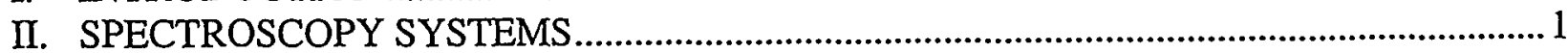

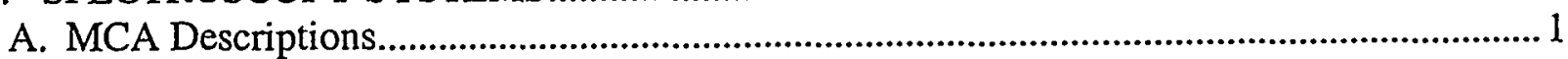

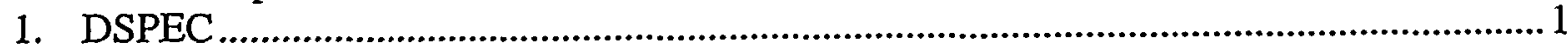

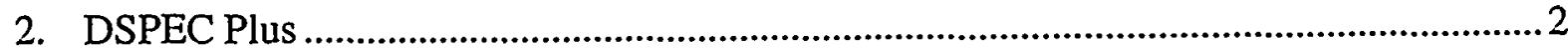

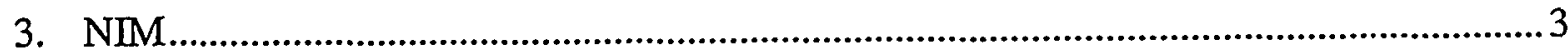

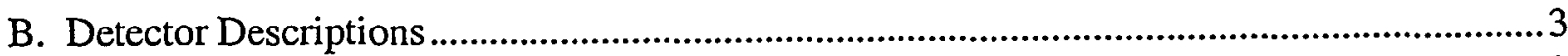

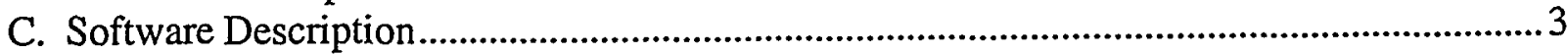

III. TESTING THE SYSTEMS .........................................................................................

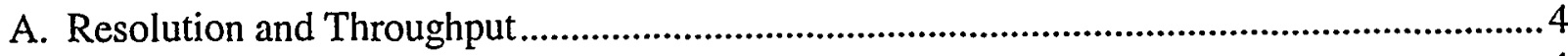

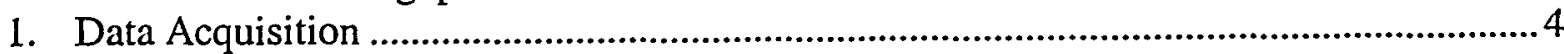

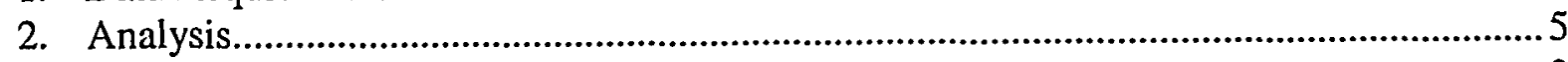

3. Comparison with Other Systems ……….................................................................... 8

B. Peak Stability.................................................................................................................... 10

1. Peak Position as Function of Input Count Rate .................................................................11

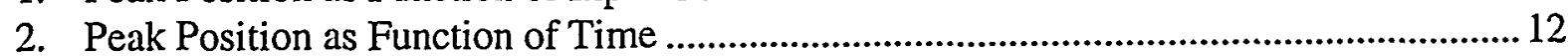

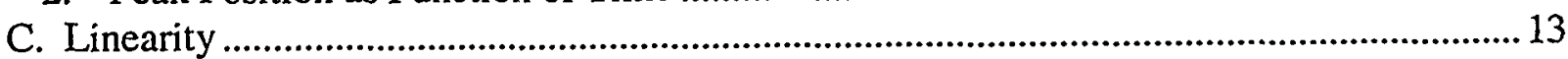

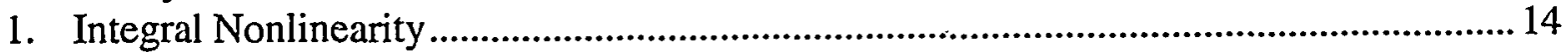

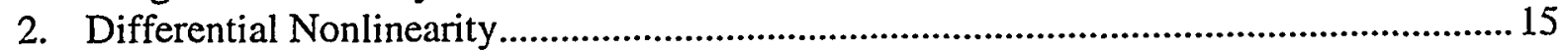

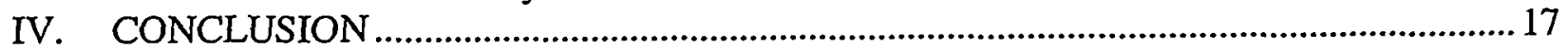

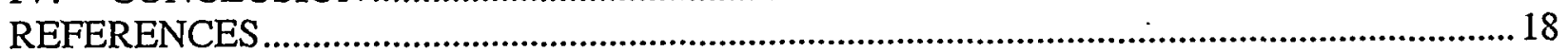




\title{
COMPARISONS OF THE DSPEC AND DSPEC PLUS SPECTROMETER SYSTEMS
}

\author{
by
}

Duc T. Vo

\begin{abstract}
A previous experimental evaluation ${ }^{1}$ of nine commercial gamma-ray spectroscopy systems demonstrated significant benefits of digital signal processing for improvements in the performance of high-resolution gamma-ray spectroscopy systems. Spectacular improvements in the energy resolution and throughput of germanium detectors 'were demonstrated for the DSPEC. Recently, a new, improved version of DSPEC, the DSPEC Plus, was developed. Results of the performance of the DSPEC Plus are compared to those of the DSPEC.
\end{abstract}

\section{INTRODUCTION}

Safeguards isotopic measurements require the best of the spectrometer systems with excellent resolution, stability, and throughput. Until recently, gamma-ray spectroscopy has always been done using the analog amplifier, which processes the pulses from the preamplifier to remove the noise, rejects the pile-up signals, and shapes the signals into some desirable forms before sending them to the analog-to-digital converter $(A D C)$ to be digitized. The digital signal processors (DSP) was introduced few years ago. Instead of processing the pulses from the preamplifier in a similar way to that of a conventional analog spectroscopy amplifier, the DSP systems process the pulses digitally. In late 1996, EG\&G Ortec introduced a Digital Gamma-Ray Spectrometer (DSPEC) that uses digital technology to analyze the preamplifiers' pulses from all types of germanium, silicon, and Nal detectors. Shortly afterward, Canberra Industry also released its version of DSP systems, the model 2060 Digital Signal Processor and the model 9660 ICB Programmable Digital Signal Processor. These DSP systems, especially the DSPEC, perform much better than any analog system when used with a germanium detector. Spectacular improvements in the energy resolution and throughput of the germanium detectors were demonstrated in Ref. 1.

In 1999, EG\&G Ortec released the latest DSP system, the DSPEC Plus, which supposes to be an improved version of DSPEC. The DSPEC Plus, the DSPEC, and the standard nuclear instrumental methods (NIM) system are tested with a coaxial and a planar germanium detectors.

\section{SPECTROSCOPY SYSTEMS}

\section{A. MCA Descriptions}

\section{DSPEC}

DSPEC is an AC-powered, stand-alone unit. Unlike the traditional signal processing where the pulses from the preamplifier are processed by the analog amplifier and then digitized by the ADC, DSPEC digitally processes the pulses directly from the preamplifier using a quasitrapezoid pulse shape. The four parameters controlling the pulse shape are rise time, cusp, flattop, and tilt. DSPEC's rise time is roughly equivalent to twice the integration time set on a 
conventional analog spectroscopy amplifier. The cusp controls the curvature of the sides of the quasi-trapezoid, and the flattop adjusts the width of the top of the quasi-trapezoid. The cusp and flattop values can be adjusted to obtain the best results for different detector, radiation source, or count rate. The tilt controls the flattop slope and the DSPEC optimizer automatically sets its value. The DSPEC's Baseline Restorer (BLR) can be set to auto, fast, slow, or manual. In this evaluation, it was set to auto. It also has a built-in "Virtual Oscilloscope" (viewed with the Maestro software from Ortec) that would eliminate the need for an external oscilloscope.

Each DSPEC processes the output of a single detector. DSPEC can be connected to the computer by means of an ethernet BNC connector, Dual-Port Memory (DPM) 37-pin D-type connector, or low-speed serial link. If there are more than one DSPEC-controlled detector, with the ethernet data link connection method, multiple systems could be setup to be controlled by one computer by chaining all the DSPECs together into a single local area network (LAN). Likewise, multiple computers can also be set using a LAN to control single or multiple DSPECs. The DSPEC can also communicate with the computer using the DPM data link. It requires a DPM interface card such as the Advance Data Collection and Management (ADCAM) interface card from Ortec that plugs into the host computer. If the DPM data link is used, it is still easy to connect up to eight DSPEC-controlled detectors or many other combinations of Ortec multichannel buffers (MCB) to one computer. A dual-port fan-out module is needed to turn the single connector on the interface card into enough connectors for the multiple systems. The serial link of the DSPEC is provided for convenient and debugging and is normally not used due to the low data-transfer speed.

\section{DSPEC Plus}

DSPEC Plus is similar to its predecessor, the DSPEC. However, there are several differences that the DSPEC Plus has improved upon its predecessor. Table I below shows the differences between the two systems.

The DSPEC Plus has more functions than the DSPEC (TTL-type remote shutdown, count rate meter) and for those functions in which both systems possess (rise time, flattop, cusp), the DSPEC Plus has more finer-step adjustments, which allow the user to fine tune the system better. Also, for the high-voltage (HV) or the amplifier polarity setup, the DSPEC Plus controls them from the computer instead of physically opening the case of the unit to change them like the DSPEC. This aspect would make it easier and more convenient to install DSPEC Plus into hardto-retrieve spaces and control it from the desktop computer.

In addition to the differences above, DSPEC Plus also features the zero dead time (ZDT) mode of operation. When DSPEC Plus operates in the ZDT mode, it makes up for the live-time losses by taking very short acquisitions and applying a correction in real time to the number of counts in the spectrum. When operating in the ZDT mode, the system stores both the corrected and uncorrected spectra. However, in the ZDT mode, the throughput of the system is reduced somewhat as extra processing must be done on the spectrum. 
Table I. Differences between DSPEC and DSPEC Plus.

\begin{tabular}{|c|c|c|}
\hline Function & DSPEC & DSPEC Plus \\
\hline Remote shutdown & Working with Ortec detector only. & $\begin{array}{l}\text { Working with both Ortec and other detectors } \\
\text { that use the TTL-type inhibit circuits such as } \\
\text { the Canberra detectors. Computer selectable. }\end{array}$ \\
\hline HV output & $\begin{array}{l}\text { Opening the case to move the internal } \\
\text { jumper board to positive HV or negative } \\
\text { HV position. }\end{array}$ & $\begin{array}{l}\text { Separate BNC connectors for positive HV } \\
\text { and negative HV. Computer selectable. }\end{array}$ \\
\hline HV bias setup & 10-turn potentiometer. & Computer control. \\
\hline Amplifier polarity & $\begin{array}{l}\text { Opening the case to switch to positive or } \\
\text { negative polarity. }\end{array}$ & Computer selectable. \\
\hline $\begin{array}{l}\text { Rise time and fall } \\
\text { time }\end{array}$ & $\begin{array}{l}32 \text { rise and fall times ranging from } 0.8 \text { to } \\
25.6 \mu \mathrm{s} \text { in } 0.8-\mu \mathrm{s} \text { steps. }\end{array}$ & $\begin{array}{l}115 \text { rise and fall times ranging from } 0.2 \text { to } \\
23.0 \mu \mathrm{s} \text { in } 0.2-\mu \mathrm{s} \text { steps. }\end{array}$ \\
\hline Flattop & $\begin{array}{l}5 \text { flattop times ranging from } 0.8 \text { to } 2.4 \\
\mu \mathrm{s} \text { in } 0.4-\mu \mathrm{s} \text { steps. }\end{array}$ & $\begin{array}{l}22 \text { flattop times ranging from } 0.3 \text { to } 2.4 \mu \mathrm{s} \text { in } \\
0.1-\mu \text { s steps. }\end{array}$ \\
\hline Cusp parameter & 6 values ranging from 0.5 to $<1$. & 7 values ranging from 0.4 to $<1$. \\
\hline Count rate meter & N/A & Available from software. \\
\hline
\end{tabular}

\section{NIM}

The NIM system was a standard system which consisted of an Ortec 4001C NIM bin power supply, Canberra 3106D HV power supply, Ortec 672 Spectroscopy Amplifier, Canberra 8077 Fast ADC, Canberra 8232 Digital Stabilizer, Ortec 996 Counter Timer, and Ortec Ethernim MatchMaker. The triangular shaping of the amplifier was used for all the data collection. The BLR was set to "auto." The PUR output from the amplifier was connected to the PUR input of the MatchMaker.

This NIM system, with the MatchMaker, can be connected to the computer in the same ways as that of the DSPEC and DSPEC Plus by means of an ethernet BNC connector, DPM 37 pin D-type connector, or low-speed serial link.

\section{B. Detector Descriptions}

Two detectors, one coaxial and one planar, were used for the evaluation. Table II lists the specifications of these detectors. The $25 \%$ coaxial detector is degraded and its performance at high energy is much worse than the values listed in Table $\Pi$.

\section{Software Description}

The software used to acquire data was Maestro v5.1 from EG\&G Ortec. In this experiment, all three systems were chained together with the IBM ThinkPad $760 \mathrm{XD}$ by ethernet through the 3Com Etherlink III PCMCIA card into a single LAN. 
Table II. Specifications of the detectors. The specified resolutions are the warranted values from the manufacturers.

\begin{tabular}{|l|l|l|}
\hline Germanium detector & Coaxial & Planar \\
\hline Manufacturer & Canberra & Ortec \\
\hline Manufacture date & Nov 1989 & Jun 1999 \\
\hline Diameter (mm) & 60.0 & 25.4 \\
\hline Length (mm) & 42.0 & 16.2 \\
\hline Relative efficiency (\%) & 25 & N/A \\
\hline FWHM @ 1.33 MeV, $6 \mu \mathrm{s}$ shaping, $1 \mathrm{kHz}$ & $1.75 \mathrm{keV}$ & N/A \\
\hline FWHM @ 1.33 MeV, $2 \mu \mathrm{s}$ shaping, $1 \mathrm{kHz}$ & $1.94 \mathrm{keV}$ & N/A \\
\hline FWHM @ 1.33 MeV, $2 \mu \mathrm{s}$ shaping, 30 kHz & $2.00 \mathrm{keV}$ & N/A \\
\hline FWHM @ 122 keV, $6 \mu \mathrm{s}$ shaping, $1 \mathrm{kHz}$ & $750 \mathrm{eV}$ & N/A \\
\hline FWHM @ 122 keV, $3 \mu \mathrm{s}$ shaping, $1 \mathrm{kHz}$ & N/A & $510 \mathrm{eV}$ \\
\hline FWHM @ 122 keV, $2 \mu \mathrm{s}$ shaping, $1 \mathrm{kHz}$ & $850 \mathrm{eV}$ & N/A \\
\hline FWHM @ 122 keV, $2 \mu \mathrm{s}$ shaping, $30 \mathrm{kHz}$ & $880 \mathrm{eV}$ & N/A \\
\hline FWHM @ 122keV, $1 \mu \mathrm{s}$ shaping, $50 \mathrm{kHz}$ & N/A & $580 \mathrm{eV}$ \\
\hline
\end{tabular}

\section{TESTING THE SYSTEMS}

\section{A. Resolution and Throughput}

\section{Data Acquisition}

The planar and the $25 \%$ coaxial germanium detectors were used in the measurements (see Table II). The detectors were shielded with lead bricks and cadmium sheets (except the fronts) to reduce the background gamma rays. Cobalt-57 (with energy peaks at 122 and $136 \mathrm{keV}$ ) and cobalt-60 (with energy peaks at 1173 and $1332 \mathrm{keV}$ ) were used. For these measurements, each source was measured separately. The sources were moved close to or far away from the detectors in order to achieve the desired count rates. When running with the coaxial detector, the ADC conversion gain and range were set at $8 \mathrm{~K}$ on all the systems. For the planar detector, the ADC conversion gain and range were set at $4 \mathrm{~K}$ on all the systems. The amplifier gains were adjusted so that for the ${ }^{57} \mathrm{Co}$ data the $122-\mathrm{keV}$ peak was at channel 3200 and for the ${ }^{60} \mathrm{Co}$ source, the $1332-\mathrm{keV}$ peak was at channel 7800 . The gain stabilizers were set on either the $122-\mathrm{keV}\left({ }^{57} \mathrm{Co}\right)$ peak or the $1332-\mathrm{keV}\left({ }^{60} \mathrm{Co}\right)$ peak. The zero stabilizers were not used.

For the planar detector, the shaping times of 1,2 , and $3 \mu$ s were used with input count rates at $3,10,20,40,60,80$, and $100 \mathrm{kHz}$. For the coaxial detector, the shaping times of 2, 3, and $6 \mu$ s were used with input count rates at 3,10,30,60, and $100 \mathrm{kHz}$. Note that the DSP systems (DSPEC and DSPEC Plus) use the rise times instead of the shaping times of the analog systems. The rise time of the DSP systems is roughly equivalent to twice the shaping time set on a conventional analog spectroscopy amplifier. For the shaping times of $1,2,3$, and $6 \mu$ s that the NIM system used in this evaluation, the DSP systems used the corresponding rise times of 2.4, $4.0,6.4$, and $12.0 \mu \mathrm{s}$, respectively. Due to the smaller rise time steps, the DSPEC Plus could use the rise times of 2.0 and 6.0 to correspond to 1 and $3 \mu$ s shaping times. However, the rise time of 2.4 and $6.4 \mu$ s were used instead so that it would be the same as that of the DSPEC. From now 
on, in this paper, whenever we mention the shaping time of $1,2,3$, or $6 \mu$ s for the DSP system, we mean the corresponding rise time of $2.4,4.0,6.4$, or $12.0 \mu \mathrm{s}$.

For the DSP systems, there are many combinations of the flattop and cusp values. The optimal combination is different for each detector. Using the wrong values or combinations would lead to bad resolution and/or throughput. There is no function or button that can be used to automatically set the flattop and cusp values for optimal performance. (The DSP systems from Canberra Industry can automatically set the flattop for optimal performance.) The user needs to find the optimal combination for a detector manually.

The method as described in Ref. 2 was used to obtain the optimal combinations for these two detectors. Table III shows the optimal combinations for the DSPEC and DSPEC Plus with the two detectors used in this evaluation.

Table III. Optimal flattop and cusp values.

\begin{tabular}{|l|l|l|l|l|}
\hline \multirow{2}{*}{} & \multicolumn{2}{|c|}{ Planar } & \multicolumn{2}{c|}{ Coaxial } \\
\cline { 2 - 5 } & Flattop & Cusp & Flattop & Cusp \\
\hline DSPEC & $1.2 \mu \mathrm{s}$ & 0.7 & $1.6 \mu \mathrm{s}$ & 0.8 \\
\hline DSPEC Plus & $0.8 \mu \mathrm{s}$ & 0.6 & $1.0 \mu \mathrm{s}$ & 0.6 \\
\hline
\end{tabular}

The optimal flattop and cusp values of the DSPEC Plus are somewhat smaller than that of the DSPEC. Note that while different cusp values have no effect on the throughput of a system, smaller flattop value would tend to increase the throughput. ${ }^{2}$ Therefore, it may be expected that the DSPEC Plus would have slightly better throughput than the DSPEC.

\section{Analysis}

The spectra were analyzed using Maestro. The energy calibrations were done using either the $122.06-\mathrm{keV}$ and $136.47-\mathrm{keV}$ peaks of ${ }^{57} \mathrm{Co}$ or the $1173.23-\mathrm{keV}$ and $1332.49-\mathrm{keV}$ peaks of ${ }^{60} \mathrm{Co}$. The net counts in the $122-\mathrm{keV}$ and $1332-\mathrm{keV}$ peaks were used to calculate the throughput rate. To ease the data viewing, the resolution results are then fitted to the equation $a+b \exp (c x)$ and the throughput results are fitted to the equation $a x \exp \left(b x^{c}\right)$ where $a, b$, and $c$ are some variables and $x$ is the input count rate. Figures 1,2 , and 3 show the fitted resolution and throughput results of all three systems with both detectors. For all the results shown in Figs. 1, 2, and 3 , the analysis was done on the $122-\mathrm{keV}$ peak of the ${ }^{57} \mathrm{Co}$ and the $1332-\mathrm{keV}$ peak of the ${ }^{60} \mathrm{Co}$.

In general, the DSP systems are better than the NIM system at both resolution and throughput performance with both detectors and both sources, especially with the coaxial detector at high energy. As for the comparison between the two DSP systems, the DSPEC Plus appears to have slightly better throughput than the DSPEC with both detectors and both sources. For the resolution, the DSPEC Plus is better with the coaxial detector at high energy $\left({ }^{60} \mathrm{Co}\right.$ source) and is somewhat worse at low energy $\left({ }^{57}\right.$ Co source).

The reason the DSPEC Plus has somewhat better throughput than the DSPEC probably due to the smaller flattop values used (see Table III). As for the somewhat worse resolution of 

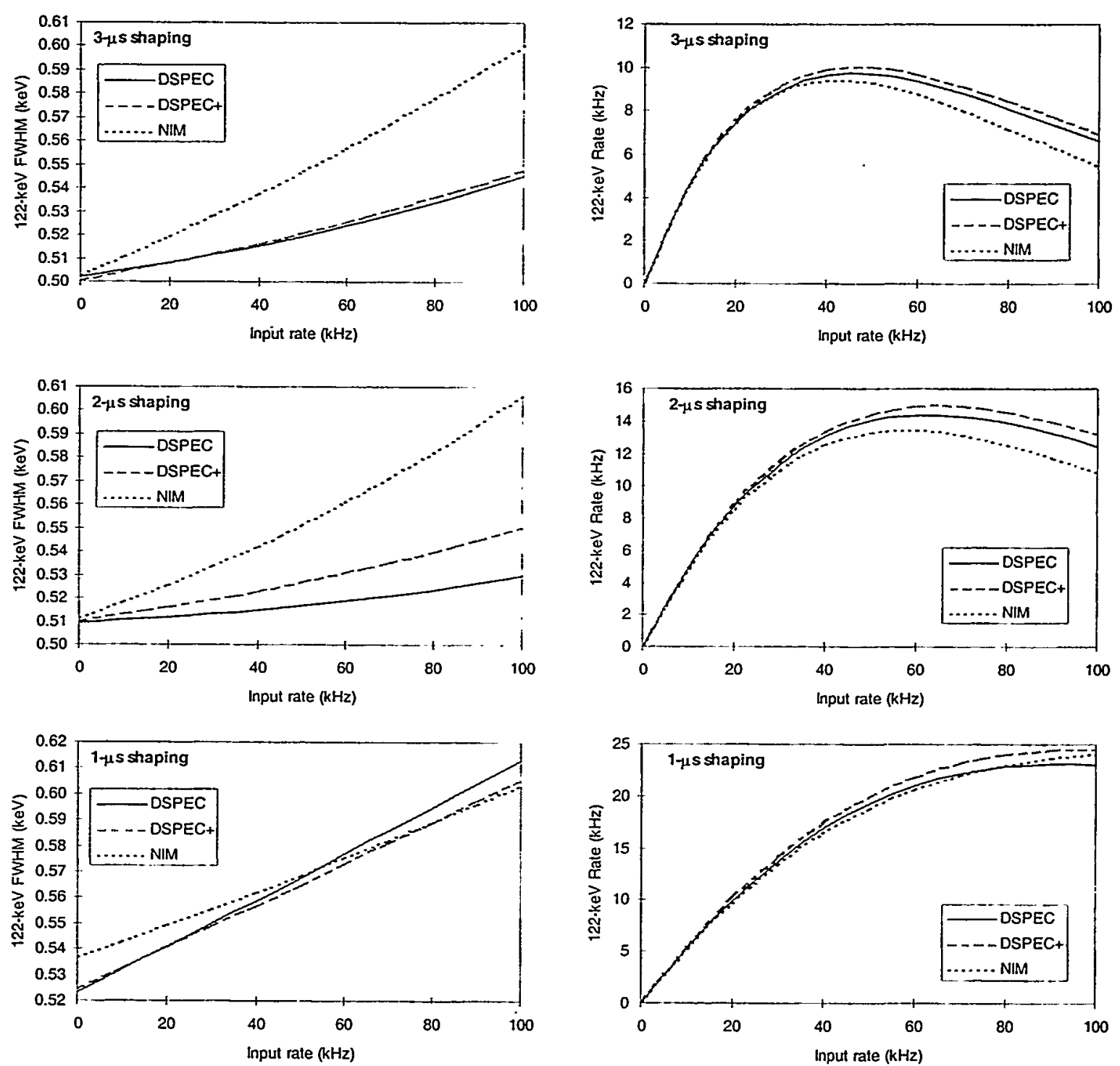

Fig. 1. Comparative resolution and throughput of three systems with the planar detector using the ${ }^{57}$ Co source.

the DSPEC Plus with the ${ }^{57}$ Co source, it may (or may not) come from the inability to optimize the system properly as described below.

During the testing of the systems, we found that the DSPEC Plus had a problem with the optimization and pole zero when the coarse gain was set at five or larger. It could properly optimize the system with coarse gain of one or two. This does not affect the data with the ${ }^{60} \mathrm{Co}$ source since for this data set, the coarse gain was set at two. However, with the ${ }^{57} \mathrm{Co}$ source, to set the 122-keV peak at channel 3200, the coarse gain had to be set at five or larger (for both the planar and the coaxial detectors). This would prevent us from properly optimizing the DSPEC 

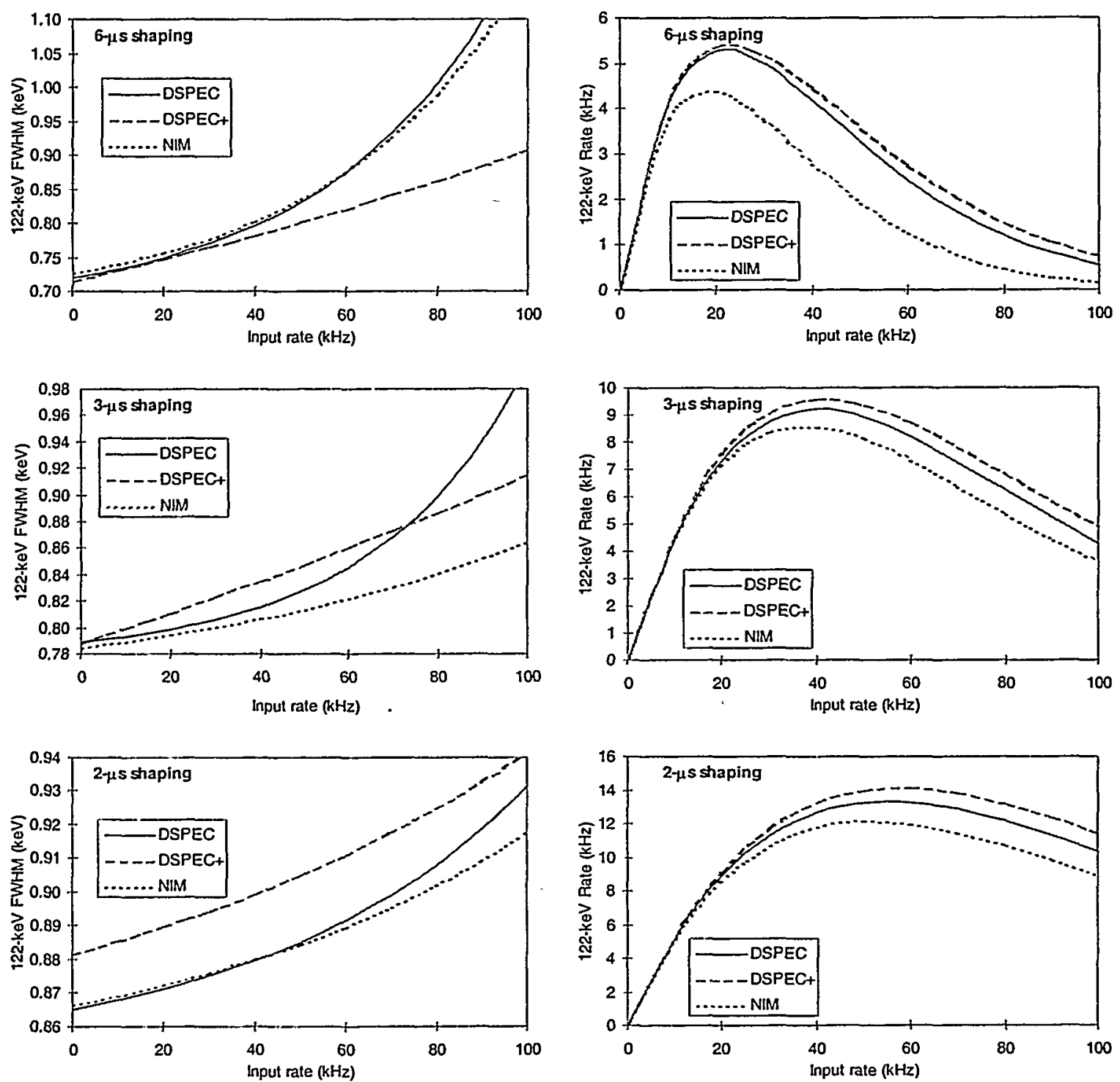

Fig. 2. Comparative resolution and throughput of three systems with the coaxial detector using the ${ }^{57} \mathrm{Co}$ source.

Plus. A trick was used by setting the coarse gain at two for the optimization. After it was optimized, the coarse and fine gains were then readjusted to shift the $122-\mathrm{keV}$ peak to the channel 3200 for the data acquisition.

This "unable to optimize" problem has since been corrected by the manufacturer. With the fixed DSPEC Plus, we took the spectra, some with the optimization done using the trick described above and some with the optimization done when the amplifier gain was about right. The results were not different. This means that the results of the DSPEC Plus with the ${ }^{57} \mathrm{Co}$ source presented in Figs. 1 and 2 are valid. 


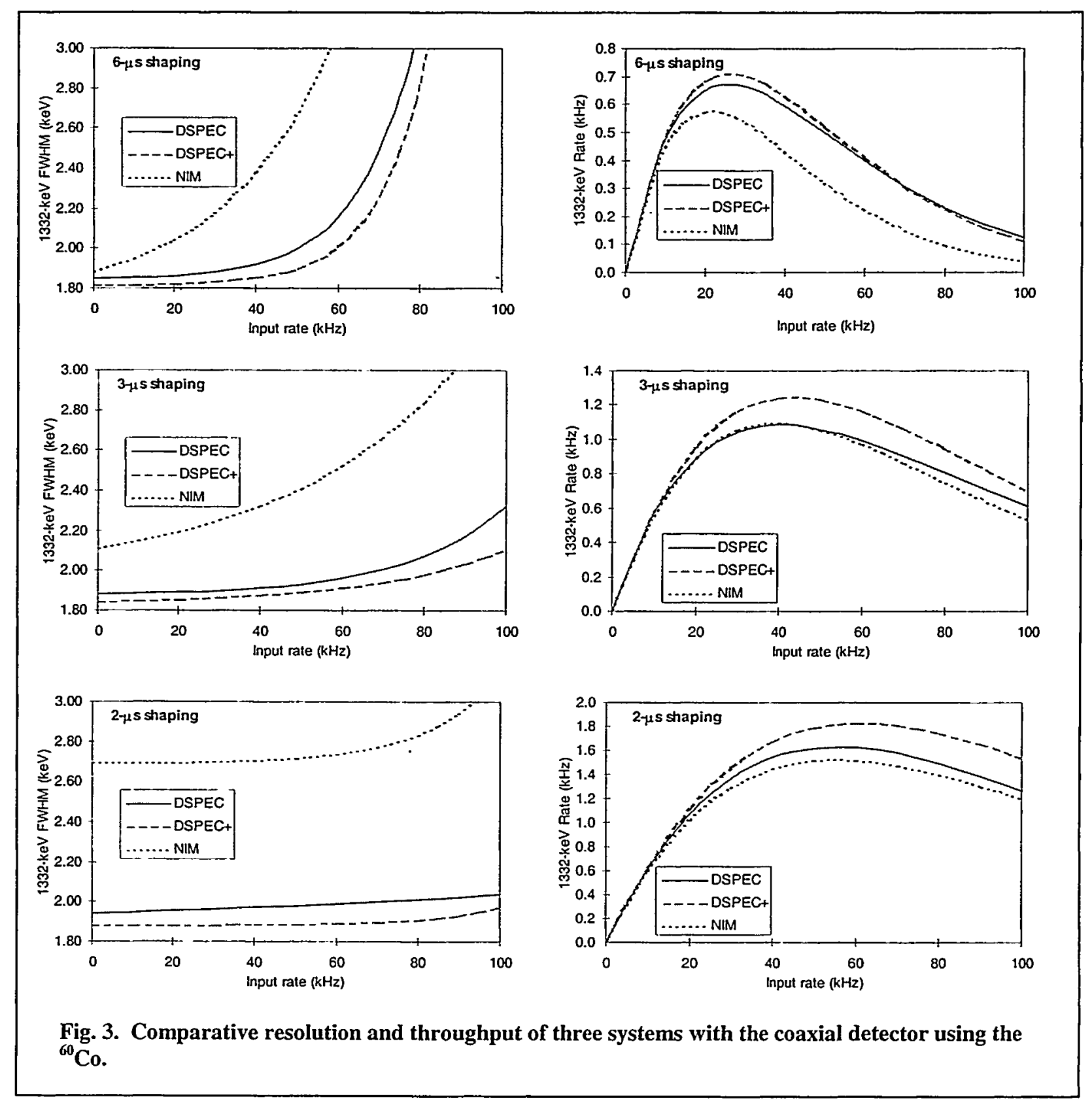

However, it is not known if the problem with the optimization we experienced before would lead to some other problems that might have affected the resolution of the DSPEC Plus. Due to the lack of time, we cannot retake all the data with the now fixed DSPEC Plus.

\section{Comparison with Other Systems}

It is of interest to compare these measurements with the archival measurements of other systems. Reference 1 lists the comparison of nine systems using various detectors. The coaxial detector that we used in this evaluation is the same as one of the detectors used in Ref. 1. Therefore, we may be able to compare the results of the coaxial detector used in this evaluation with the results of the same coaxial detector used in Ref. 1. 
In Ref. 1, the data for this coaxial detector were taken with 2-, 4-, and 6- $\mu$ s shaping. In this evaluation, 2-, 3-, and 6 - $\mu$ s shaping times were used. So only the results with the shaping times of 2 and $6 \mu$ s are compared.

Also, this detector was somewhat degraded at the time it was used for acquiring data in Ref. 1. At the time we took data for this evaluation, it was further degraded. Visually checking the two sets of results, the resolution at low energy $\left(122 \mathrm{kcV}\right.$ of $\left.{ }^{57} \mathrm{Co}\right)$ in this evaluation was only slightly worse than that in Ref. 1 . However, at high encrgy $\left(1332 \mathrm{keV}\right.$ of $\left.{ }^{60} \mathrm{Co}\right)$, it is about $7 \%$ worse. Therefore, to accurately compare the two sets of results, one of them needs to be normalized to the other.

To normalize the resolution results of this evaluation to those in Ref. 1, the results of the coaxial detector shown in Figs. 2 and 3 are then multiplied by some constants such that the curves of the DSPEC and the NIM match the data points of the DSPEC and the NIM in Ref. 1. These normalization constants are then used to normalize the results of the DSPEC, DSPEC Plus, and NIM in this evaluation to those from Ref. 1.

The normalized comparisons with both the ${ }^{57} \mathrm{Co}$ and ${ }^{60} \mathrm{Co}$ at 2- and 6- $\mu$ s shaping times of the DSPEC, DSPEC Plus, and NIM are shown in Figs. 4 and 5 together with the results for seven other systems taken from Ref. 1. The seven other systems are the Dart, Nomad Plus, and 92X-II

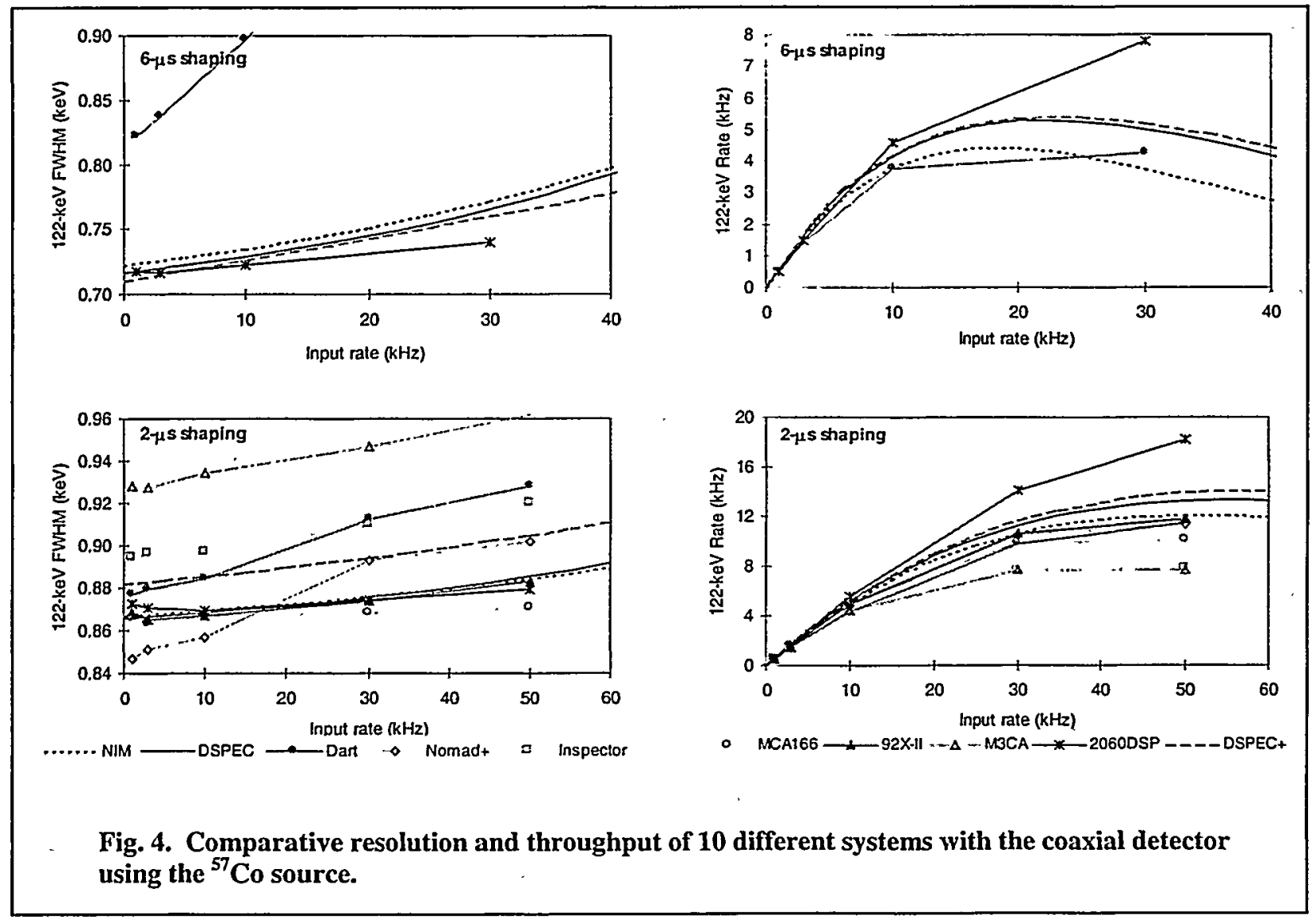




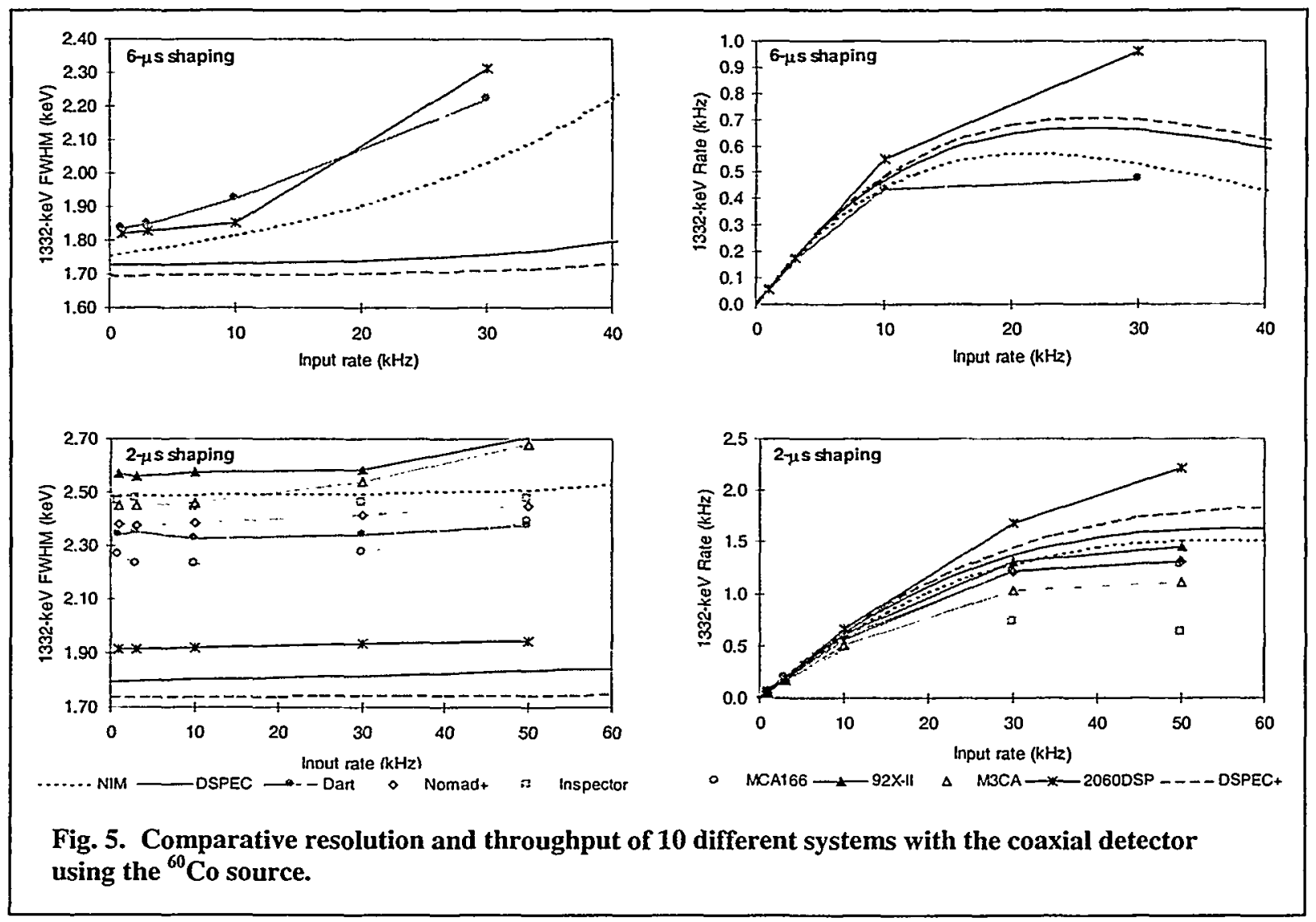

from Ortec, Inspector and model 2060 Digital Signal Processor (2060DSP) from Canberra, MCA166 from GBS-Elecktronik, and the M3CA from Aquila Technologies. In these figures, the results from the three systems used in this evaluation are plotted as smooth curves to distinguish them from the other systems.

Note the excellent performance of the DSP systems comparing with the analog systems, especially at high energy with small shaping time. It is because this coaxial detector is degraded and it displays large ballistic deficit effect. (Ballistic deficit occurs when the charge collection time of the detector is too long and the amplifier is unable to completely shape and filter the pulses from the detector. This effect is shown more clearly with large detectors and large energy.) The digital signal-processing circuits of the DSP systems can minimize the ballistic deficit much more effectively then the analog systems, which results in much better resolution.

\section{B. Peak Stability}

It is of interest to study the peak stability of these systems as a function of input count rate and as the long-term drift where the ambient temperature may change over a long time period. Table IV shows the specifications of the three systems. The NIM system uses both the Ortec 672 amplifier and the Canberra $8706 \mathrm{ADC}$. Therefore, the specifications of the NIM equal the quadrature sum of these two modules. The "peak shift" term is specified for input rate up to $100 \mathrm{kHz}$. For the "zero shift," the amplifier specifies it as $7.5 \mu \mathrm{V} /{ }^{\circ} \mathrm{C}$. The value in the table is converted to percent per Celsius degree, assuming the amplifier gain is about 10 and the full voltage output is $10 \mathrm{~V}$. 
Table IV. Specifications for the peak stability of the three systems.

\begin{tabular}{|l|c|c|c|c|c|}
\hline & $\begin{array}{c}\text { DSPEC } \\
\text { DSPEC Plus }\end{array}$ & $\begin{array}{c}\text { Ortec 672 } \\
\text { Amplifier }\end{array}$ & $\begin{array}{c}\text { Canberra 8706 } \\
\text { ADC }\end{array}$ & NIM \\
\hline Peak shift $(\%)$ & N/A & N/A & $<0.018$ & $<0.025$ & $<0.03$ \\
\hline Gain shift $\left(\% /{ }^{\circ} \mathrm{C}\right)$ & $<0.005$ & $<0.005$ & $<0.005$ & $<0.009$ & $<0.01$ \\
\hline Zero shift $\left(\% /{ }^{\circ} \mathrm{C}\right)$ & $<0.001$ & $<0.001$ & $<0.0008$ & $<0.0025$ & $<0.0026$ \\
\hline
\end{tabular}

\section{Peak Position as Function of Input Count Rate}

The coaxial detector was used for this test. The ${ }^{57} \mathrm{Co}$ and ${ }^{60} \mathrm{Co}$ sources were used separately to determine the shifting of the peaks as the input count rate gradually increased. The peak positions were recorded at count rates of $1,3,10,20,40,60,80$, and $100 \mathrm{kHz}$. The shaping time was $2 \mu \mathrm{s}$. No stabilizers were used to stabilize the peaks. For the ${ }^{57} \mathrm{Co}$ source, the $122-\mathrm{keV}$ peak was started at about channel 3200 and for the ${ }^{60} \mathrm{Co}$ source, the $1332-\mathrm{keV}$ peak was started at about channel 7800 .

Figure 6 shows the variation of the positions of the 122- and 1332-keV peaks as absolute channel position. Figure 7 shows the variation of the positions of the peaks as fractional deviation from the first ones. The overall performance of the two DSP systems appears to be about twice better than that of the NIM system. For up to $100 \mathrm{kHz}$, all three systems shifted less than $0.1 \%$. Since the DSP systems do not have the specification for the peak shift as function of input count rate, we cannot readily say if their results in Figs. 6 and 7 are good or not. However, one may note that their peak shifts of about $0.04 \%$ are larger than the specified peak shift of the NIM, which is $0.03 \%$.
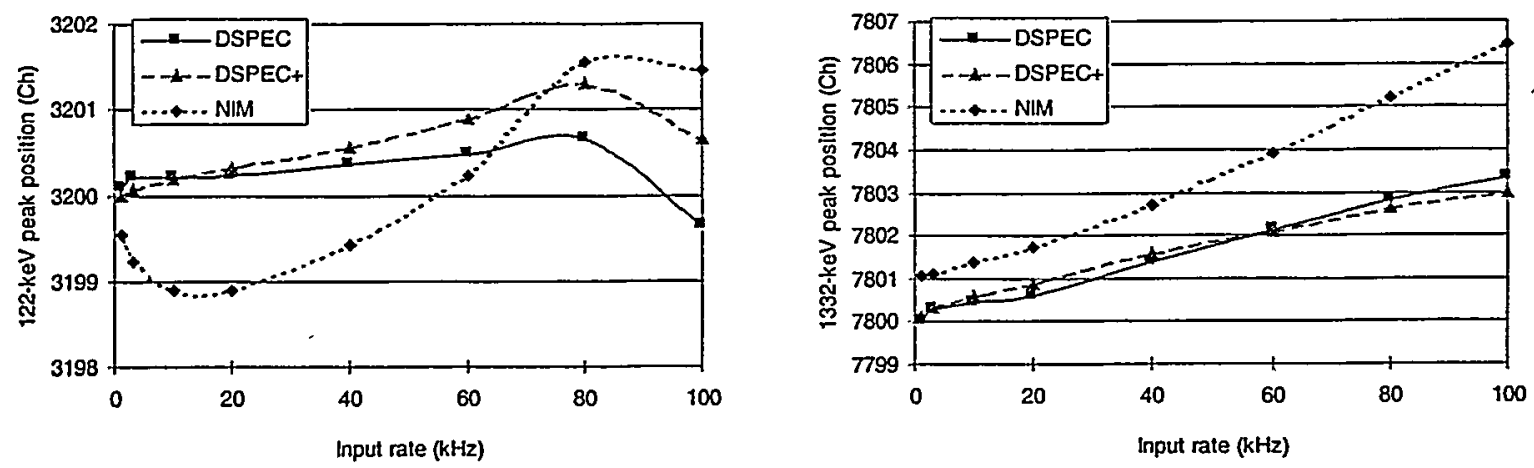

Fig. 6. The changes in peak positions of the $122-\mathrm{keV}$ peak of ${ }^{57} \mathrm{Co}$ and $1332-\mathrm{keV}$ peak of ${ }^{60} \mathrm{Co}$. 

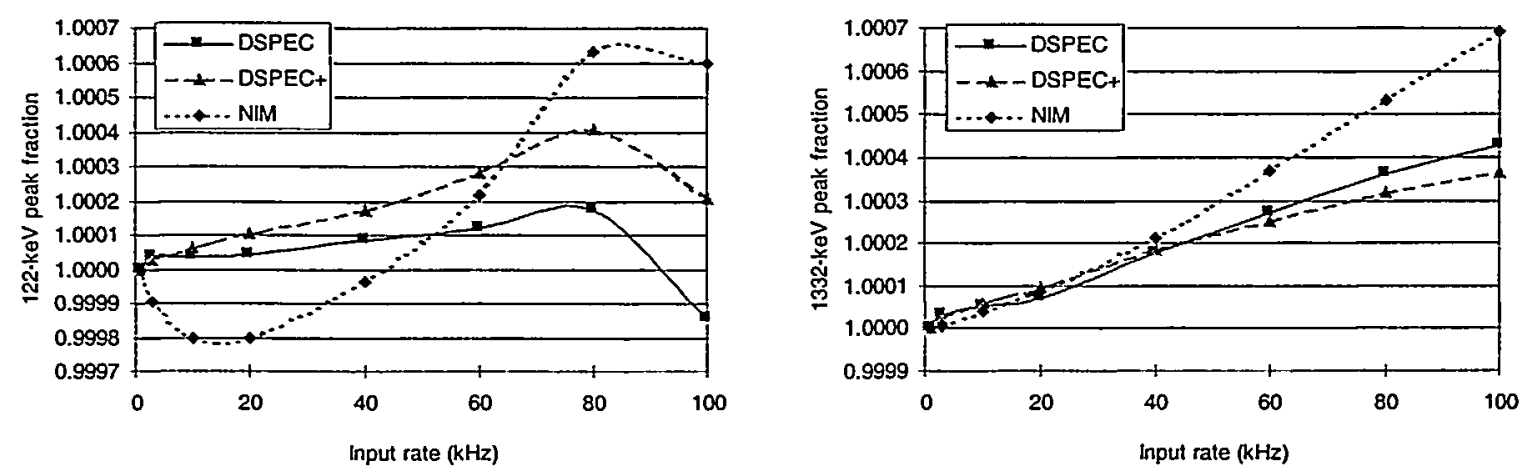

Fig. 7. The changes in peak positions of the $122-\mathrm{keV}$ peak of ${ }^{57} \mathrm{Co}$ and $1332-\mathrm{keV}$ peak of ${ }^{60} \mathrm{Co}$, expressed as fractional variation from the original position.

As for the NIM system, both the low- and high-energy peaks shift by more than $0.06 \%$, which is about twice the specified value. The reason for this is unknown but it could be that these modules (amplifier and ADC) are old and may not perform as specified.

\section{Peak Position as Function of Time}

- The coaxial detector was used for this test. The ${ }^{57} \mathrm{Co}$ and ${ }^{60} \mathrm{Co}$ sources were used together to determine the shifting of the peaks. No stabilizers were used to stabilize the peaks. The 1332$\mathrm{keV}$ peak of the ${ }^{60} \mathrm{Co}$ was set at about channel 7800 . That would put the $122-\mathrm{keV}$ peak of ${ }^{57} \mathrm{Co}$ at about channel 715 . The systems were set up to collect and store the data every 30 minutes. For each system, 40 spectra were recorded in a 20 -hour period. Those 40 spectra from each system were then summed together to form the total spectra. To obtain the changes in peak positions of the 122- and 1332-keV peaks as the fractional variation from the average, the peak positions of the individual spectra were then divided by the peak positions of the total spectrum.

Figure 8 shows the variation of the positions of the peaks as fractional deviation from the average peaks. The starting time was at about $4 \mathrm{PM}$ and the ending time was at about noon the
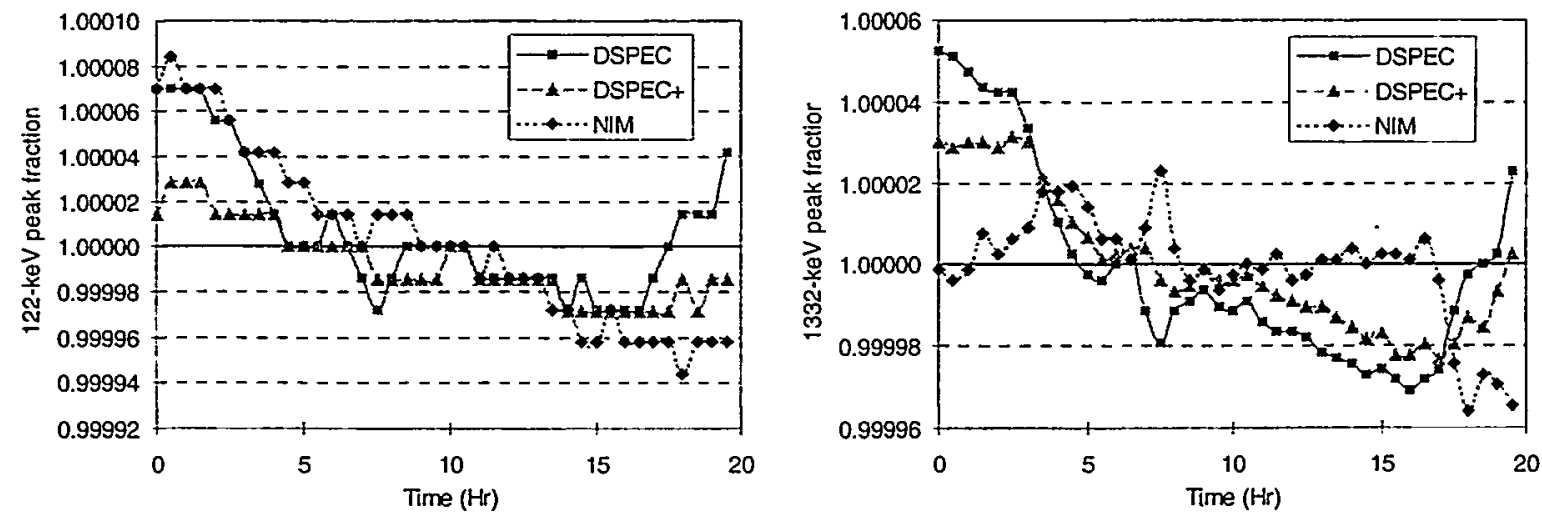

Fig. 8. The changes in peak positions of the $122-\mathrm{keV}$ peak of ${ }^{57} \mathrm{Co}$ and $1332-\mathrm{keV}$ peak of ${ }^{60} \mathrm{Co}$ as a function of time, expressed as fractional variation from the position of the peaks in the sum spectra. 
next day. The temperature in the room where the experiment was running was estimated to change by less than $2^{\circ} \mathrm{C}$ during that time period.

The DSPEC Plus appears to be the best with the peaks shifted by only about half the magnitudes of the other two systems. Overall, all three systems appear to be within their specifications as shown in Table IV.

During a long data acquisition time, how much would these peak shifts hurt the resolutions of these systems? There is apparently no effect. Table V shows the resolutions of the peaks in the sum spectra and the averages and standard deviations of the peaks in the 40 individual spectra. For each system, the peaks in the 20-hour sum spectrum appear to have the same resolution as the peaks in the 30-minute spectra. Note the differences between the resolutions of the sum spectra and the average resolutions are well within their respective standard deviations. Thus, for these three systems, the long-term data acquisition would not have any effect on the resolution of the systems.

Table V. The effect of the acquisition time on the resolutions of the systems. The values are for the FWHMs in unit of keV. "Sum" represents the 20-hour sum spectrum. "Ave." and "Stdev." stand for the average peak resolution and standard deviation of the $\mathbf{4 0}$ individual spectra.

\begin{tabular}{|l|c|c|c|c|c|c|c|c|c|}
\hline & \multicolumn{3}{|c|}{ DSPEC } & \multicolumn{3}{c|}{ DSPEC Plus } & \multicolumn{3}{c|}{ NIM } \\
\cline { 2 - 10 } & Sum & Ave. & Stdev. & Sum & Ave. & Stdev. & Sum & Ave. & Stdev. \\
\hline $122-\mathrm{keV}$ peak & 0.9004 & 0.9005 & 0.0012 & 0.9088 & 0.9087 & 0.0012 & 0.8971 & 0.8970 & 0.0012 \\
\hline $1332-\mathrm{keV}$ peak & 1.9487 & 1.9489 & 0.0059 & 1.8892 & 1.8884 & 0.0065 & 2.6899 & 2.6893 & 0.0108 \\
\hline
\end{tabular}

\section{Linearity}

The three systems are expected to be highly linear over most of their dynamic range. It is of interest to measure the nonlinearities of these systems. The maximum range of these systems is $16 \mathrm{k}$ channels. However, for some unknown reason, the MatchMaker cannot display the full $16 \mathrm{k}$-channel range of the $8077 \mathrm{ADC}$. The channels above $8 \mathrm{k}$ are wrapped. Therefore, a different ADC, model 8706 ADC (also from Canberra), was used in place of the 8077 ADC for the nonlinearity tests. Table VI shows the specified values for the nonlinearities of these systems. The values for the NIM system are the combinations of both the amplifier and the ADC.

Table VI. Nonlinearity specifications of the systems.

\begin{tabular}{|l|c|c|c|c|c|}
\hline & DSPEC & DSPEC Plus & $\begin{array}{c}\text { Ortec 672 } \\
\text { Amplifier }\end{array}$ & $\begin{array}{c}\text { Canberra } \\
8077 \mathrm{ADC}\end{array}$ & NIM \\
\hline Integral nonlinearity (\%) & $<0.025$ & $<0.025$ & $<0.025$ & $<0.025$ & $<0.035$ \\
\hline Differential nonlinearity (\%) & $<1$ & $<1$ & N/A & $<0.7$ & $<0.7$ \\
\hline
\end{tabular}




\section{Integral Nonlinearity}

Integral nonlinearity refers to the deviation from the straight line representing the relationship between input pulse height (or energy) and the output spectrum channel. To test the integral nonlinearity of a system, inputs of accurately known amplitudes are sent to the system and are plotted against the output channel assigned by the system. A straight line is then fitted to the points. The maximum deviation of the data points over the whole range of channels is taken to be the integral nonlinearity.

The input signal can be from any source. In this work, the integral nonlinearity is tested using the pulses from a precision pulser and the germanium coaxial detector. Note that, when a system are tested with the pulser, the nonlinearity of that system also includes the nonlinearity of the pulser and when it is tested with the germanium detector, its nonlinearity also includes those from the detector, the preamplifier, and the peak encrgies of the sources.

\section{a. Test with the Precision Pulser}

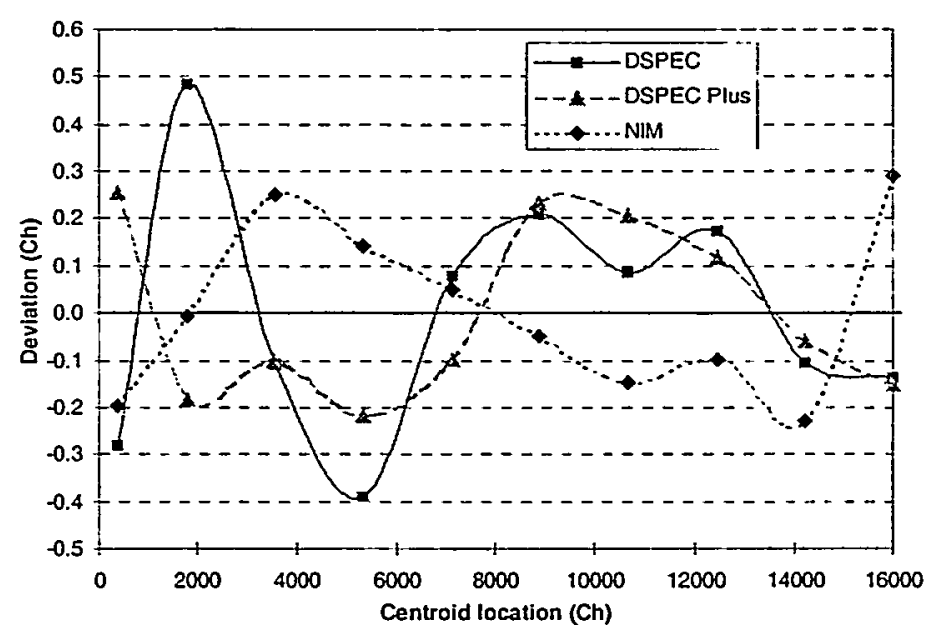

Fig. 9. Results of the integral nonlinearity tests using the pulser.
The three systems were tested using the Ortec model 448 pulser. The $2-\mu \mathrm{s}$ shaping time was used on all three systems. Figure 9 shows the results.

The results are excellent for all three systems. Even the DSPEC, which is about a factor of two worse than the other two systems, with the maximum deviation of 0.5 channels of 16,000 or $0.003 \%$ is still much less than the specification of $0.025 \%$.

\section{b. Test with the Germanium Detector}

The systems were also tested with the germanium coaxial detector. It is perhaps the better test than the test with the pulser because, after all, it tests the linearity of the entire system (consisting of detector, preamplifier, amplifier and $\mathrm{ADC}$ ). The ${ }^{133} \mathrm{Ba}$ and ${ }^{152} \mathrm{Eu}$ sources provide many gamma rays ranging from $53 \mathrm{keV}$ up to $1408 \mathrm{keV}$ to cover most of the 16,000 -channel range of the systems. Figure 10 shows the results of all three systems. 


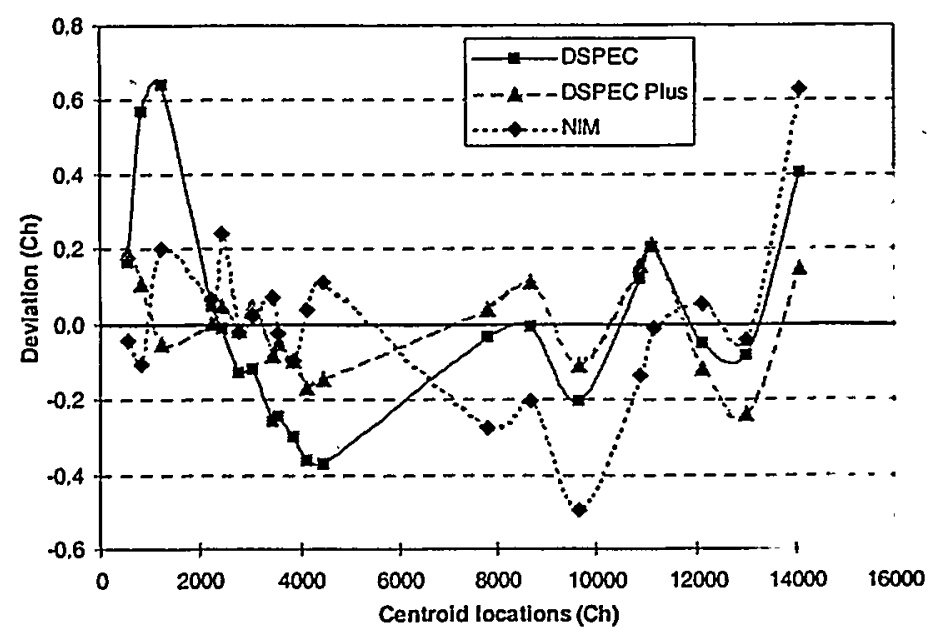

Fig. 10. Results of the integral nonlinearity tests using the coaxial germanium detector and the ${ }^{133} \mathrm{Ba}$ and ${ }^{152} \mathrm{Eu}$ sources.
All three systems perform excellently in this test too (less than $0.006 \%$ ), though not as good as with the precision pulser test. It is because this test would include the nonlinearity of the detector, preamplifier, and the accepted peak cnergies of the sources. Note the similar shape with all the peaks and valleys of all three curves in the figure. The detector, the prcamplifier, or the energies of the peaks probably cause these. Typically, the nonlinearity of a germanium and preamplifier combination is much smaller than that from the amplifier and $\mathrm{ADC}$ so one may not see them. The reason these appear in this figure is due to

the very linear of these three systems. If a system with the nonlinearity of about $0.025 \%$ (like that specified in the specifications in Table VI above) is used with this coaxial detector, one may not see the nonlinearity due to the detector and preamplifier because it will be masked by that of the amplifier and ADC.

\section{Differential Nonlinearity}

Differential nonlinearity is a measure of the equality of channel widths in an MCA system. If a perfectly flat spectrum of random pulses were presented to a perfect $A D C$ and if the were no differential nonlinearity, then every channel would have the same number of counts within the statistical uncertainty of the channel counts. We don't have a source that can provide a perfectly flat spectrum of random pulses, so we resorted to use a not-so-perfect but acceptable source to provide the pulses for this test.

In this test, a 1-in. diameter and 1-in. thick NaI detector is used with the ${ }^{137} \mathrm{Cs}$ source to provide a smooth spectrum to test the differential nonlinearity. Only the Compton continuum was included in the spectrum. The data were continuously acquired for a total of six days. The spectra from the three systems have from $\sim 130,000$ counts per channel near the end of the spectra and $\sim 3,000,000$ counts per channel at the $662-\mathrm{keV}$ back-scattered bump. The statistical uncertainty of each channel would then be limited to $\sim 0.3 \%$ for the channels near the end and $\sim 0.06 \%$ for the channels near the $662-\mathrm{keV}$ back-scattered peak. These should be sufficient for the test since the differential nonlinearity specifications of these systems are much larger (see Table VI).

The smooth spectra were obtained by smoothing the data using the 201-point cubic fit. That is, for each point on the spectra, a cubic is fitted through 201 data points centered on that point ( 100 points below and 100 points above). The smooth data point is the centered point from the fit. The smooth spectra were examined for the structures that might arise from the smoothing but none was observed. 


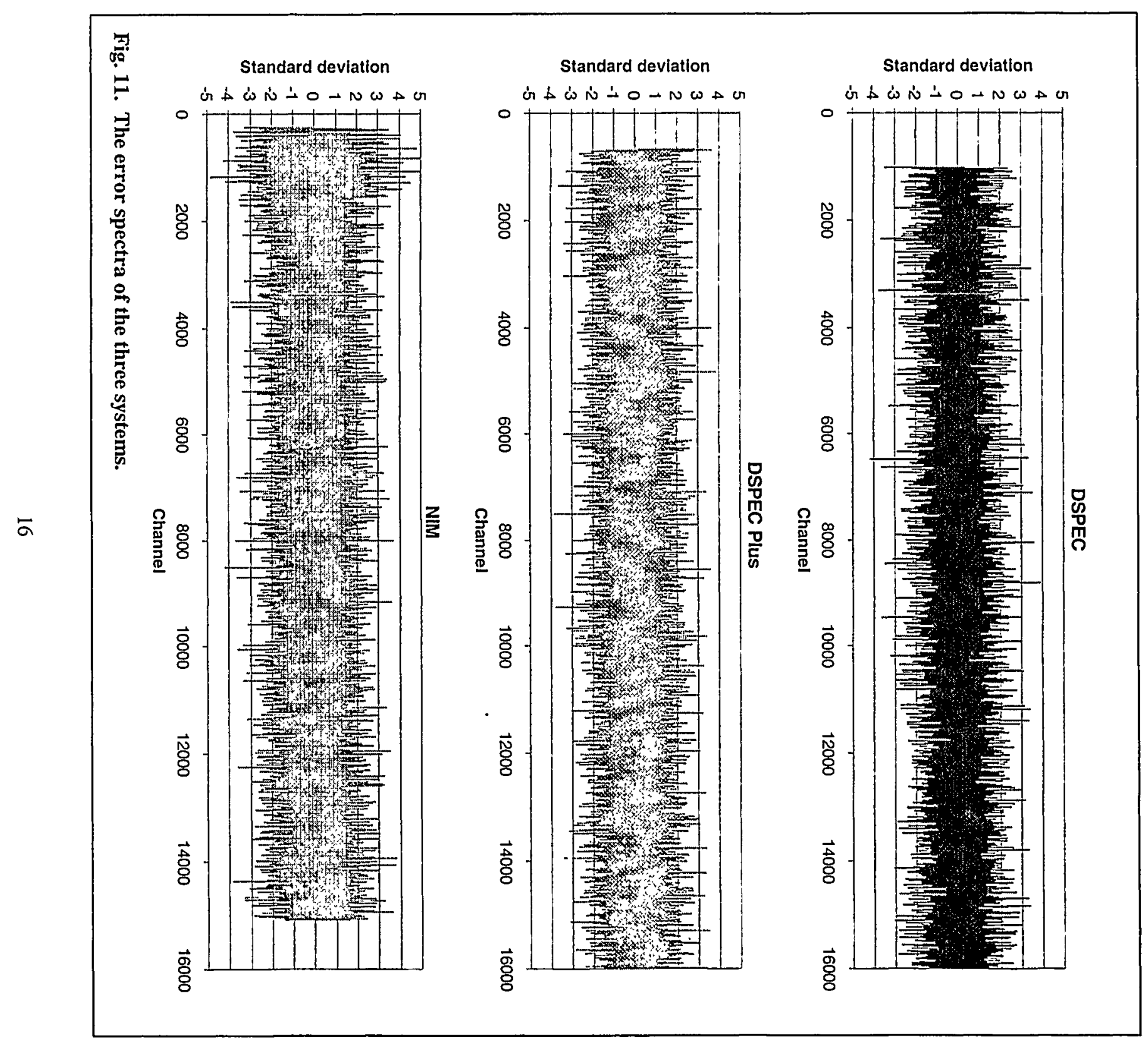

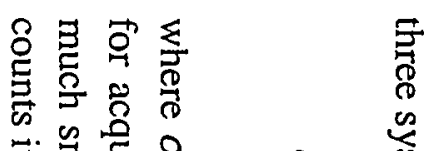

ヨ.

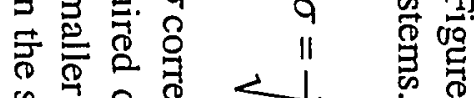

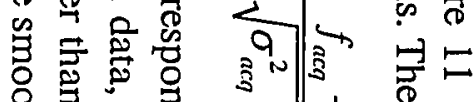

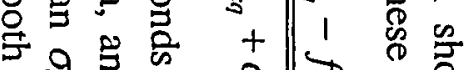

कू वे के

है

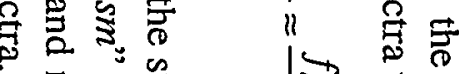

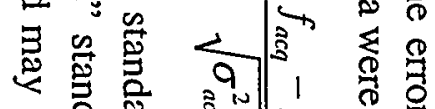

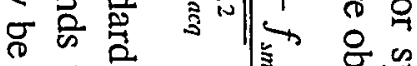

宊宫量 "

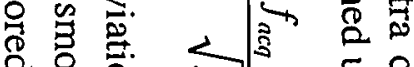

웅

2.

a

喧

总夆

的夆

용

苋总衰

2.5

के

蛋

点产言

둥

容

열. 管

蒙.

후웜

串.

氙

영

$\vec{\nabla}$

帚

苟

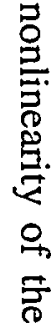


The advantage of plotting the error spectra instead of the difference spectra is that the results do not depend on the number of counts, and one can readily compare the deviation due to the differential nonlinearity to those from statistics.

Examining these spectra, one can see that most of the deviations appear to come from statistics. As for the NIM system where there appear quite a few channels with error larger than four standard deviations in the 1000-channel region, it is still much less than the specified value of $0.7 \%$ differential nonlinearity in Table VI. (For the NIM system in this 1000-channel region, one standard deviation would correspond to a deviation of $0.07 \%$.)

From these spectra, the number of the channels with the results below 1, 2, 3, and 4 standard deviations were obtained. These in turn were then compared to the theoretical statistic. Table VII shows the results. The overall results of the two DSP systems appear to completely agree with pure statistical results (i.e., the differential nonlinearity of these two systems are negligible comparing with the statistical variations which in this case vary from $\sim 0.06 \%$ for low channel to $\sim 0.3 \%$ for high channel). The overall results of the NIM are slightly smaller than from pure statistics. This means that the differential nonlinearity of the NIM is not negligible comparing with the statistics. However, they are still much below the value of $0.7 \%$ specified in Table VI.

Table VII. Fraction below the 1, 2,3, and 4 standard deviation of the three systems comparing with those from pure statistics.

\begin{tabular}{|l|c|c|c|c|}
\hline & 1 Std. Dev. & 2 Std. Dev. & 3 Std. Dev. & 4 Std. Dev. \\
\hline Statistic & 0.68268 & 0.95450 & 0.99730 & 0.99994 \\
\hline DSPEC & 0.68761 & 0.95585 & 0.99777 & 0.99993 \\
\hline DSPEC Plus & 0.68776 & 0.95593 & 0.99718 & 1.00000 \\
\hline NIM & 0.63188 & 0.92403 & 0.98996 & 0.99912 \\
\hline
\end{tabular}

\section{CONCLUSION}

Three systems were tested for the resolution and throughput performance as a function of input count rate, the stability as a function of input count rate and of long-term acquisition, and nonlinearity.

For the resolution and throughput performance, both the DSP systems are much better than the NIM system, especially when using with the coaxial detector using the ${ }^{60}$ Co source. The performance of the DSPEC Plus appears to exceed that of the DSPEC by a small but measurable amount.

For the stability performance as a function of input count rate, the two DSP systems appear to be twice better than that of the NIM system. As for the stability as a function of time (long-term acquisition), all three systems perform better than their specifications. Also, the DSPEC Plus appears to be about a factor of two better than the other two systems.

For the integral nonlinearity, all three systems perform excellently and the DSPEC Plus and NIM are about twice better than the DSPEC. 
And finally, for the differential nonlinearity, both the DSP systems seem to have negligible differential nonlinearity, while the NIM system appears to possess some.

Overall, the DSP systems have been shown to outperform the NIM system, which is about the best analog system today. Between the two DSP systems, the DSPEC Plus is somewhat better than the DSPEC in most of the categories. It is highly recommended for use in simple gamma-ray acquisition systems using germanium detectors.

\section{REFERENCES}

1. D. T. Vo, "Extended Evaluations of the Commercial Spectrometer Systems for Safeguards Applications," Los Alamos National Laboratory report LA-13604-MS (August 1999).

2. D. T. Vo, P. A. Russo, and T. E. Sampson, "Comparisons Between Digital Gamma-Ray Spectrometer (DSPEC) and Standard Nuclear Instrumentation Methods (NIM) Systems," Los Alamos National Laboratory report LA-13393-MS (October 1997). 